This document is the accepted manuscript version of the following article: Stan, C. A., Milathianaki, D., Laksmono, H., Sierra, R. G., McQueen, T. A., Messerschmidt, M., ... Boutet, S. (2016). Liquid explosions induced by X-ray 1aser pulses. Nature Physics, 12(10), 966-971. https://doi.org/10.1038/NPHYS3779

\title{
Liquid explosions induced by X-ray laser pulses
}

Claudiu A. Stan, ${ }^{1 *}$ Despina Milathianaki, ${ }^{2}$ Hartawan Laksmono, ${ }^{1}$ Raymond G. Sierra, ${ }^{1}$ Trevor A. McQueen, ${ }^{3}$ Marc Messerschmidt, ${ }^{2 \dagger}$ Garth J. Williams, ${ }^{2 \ddagger}$ Jason E. Koglin, ${ }^{2}$ Thomas J. Lane, ${ }^{2}$ Matt J. Hayes, ${ }^{2}$ Serge A. H. Guillet, ${ }^{2}$ Mengning Liang, ${ }^{2}$ Andrew L. Aquila, ${ }^{2}$ Philip R. Willmott, ${ }^{2,4}$ Joseph S. Robinson, ${ }^{2}$ Karl L. Gumerlock, ${ }^{2}$ Sabine Botha, ${ }^{5}{ }^{\S}$ Karol Nass, ${ }^{5}$ Ilme Schlichting, Robert L. Shoeman, ${ }^{5}$ Howard A. Stone, ${ }^{6}$ and Sébastien Boutet ${ }^{2}$

${ }^{1}$ Stanford PULSE Institute, Menlo Park, CA 94025, USA

${ }^{2}$ Linac Coherent Light Source, SLAC National Accelerator Laboratory, Menlo Park, CA 94025, USA

${ }^{3} S U N C A T$ Center for Interface Science and Catalysis, SLAC National Accelerator Laboratory, Menlo

${ }^{4}$ Paul Scherrer Institute, CH-5232 Villigen, Switzerland

${ }^{5}$ Max-Planck-Institut für medizinische Forschung, 69120 Heidelberg, Germany

${ }^{6}$ Department of Mechanical and Aerospace Engineering, Princeton University, Princeton, NJ 08544, USA

†Present address: National Science Foundation BioXFEL Science and Technology Center, Buffalo, NY

Present address: Brookhaven National Laboratory, Upton, NY 11973, USA

${ }^{\S}$ Present address: Department of Chemistry, Hamburg University, 20146 Hamburg, Germany

Email: cstan@slac.stanford.edu

\section{Author manuscript (copy of last submitted version, with formatting changes)}

The journal version, and the supplementary information are available online at:

http://dx.doi.org/10.1038/nphys3779

\section{Citation:}

Claudiu A. Stan, Despina Milathianaki, Hartawan Laksmono, Raymond G. Sierra, Trevor A.

McQueen, Marc Messerschmidt, Garth J. Williams, Jason E. Koglin, Thomas J. Lane, Matt J.

Hayes, Serge A. H. Guillet, Mengning Liang, Andrew L. Aquila, Philip R. Willmott, Joseph S.

Robinson, Karl L. Gumerlock, Sabine Botha, Karol Nass, Ilme Schlichting, Robert L. Shoeman,

Howard A. Stone, and Sébastien Boutet. Liquid explosions induced by X-ray laser pulses. Nature

Physics (2016). DOI: 10.1038/nphys3779 


\begin{abstract}
Explosions are spectacular and intriguing phenomena that expose the dynamics of matter under extreme conditions. We investigated, using time-resolved imaging, explosions induced by ultraintense X-ray laser pulses in water drops and jets. Our observations revealed an explosive vaporization followed by high-velocity interacting flows of liquid and vapour, and by the generation of shock trains in the liquid jets. These flows are different from those previously observed in laser ablation, due to a simpler spatial pattern of X-ray absorption. We show that the explosion dynamics in our experiments is consistent with a redistribution of absorbed energy, mediated by a pressure or shock wave in the liquid, and we model the effects of explosions including their adverse impact on X-ray laser experiments. X-ray laser explosions have predictable dynamics that may prove useful for controlling the state of pure liquids over broad energy and time scales, and for triggering pressure-sensitive molecular dynamics in solutions.
\end{abstract}




\section{(Introduction)}

Explosions are encountered in a wide range of natural and man-made systems. From supernovae at astrophysical scales, ${ }^{1}$ to the laser-induced microexplosions that are used in laser surgery, ${ }^{2}$ explosions generate high energy densities that transform rapidly into heat and kinetic energy. These processes can provide unique insights into the properties and dynamics of matter under extreme conditions. ${ }^{3,4}$

To study the release of high energy densities, explosions can be produced safely and conveniently by illuminating microscopic volumes of matter with high-energy laser pulses. ${ }^{4,5} \mathrm{An}$ example of such a process is the laser ablation of liquid water, ${ }^{6-15}$ which is an important and interesting system in its own right, but is also relevant to applications of ablation since bulk water is used as a model of soft biological tissue in laser surgery. ${ }^{2,16}$ Laser ablation in bulk water includes separable and quantifiable processes of nonlinear optical absorption, formation of plasma bubbles, and generation of shock waves. ${ }^{6-8}$ Also, laser ablation in micron-sized drops and jets of water can be used to study chemistry at high temperatures and pressures, ${ }^{9}$ or to generate extreme ultraviolet light. ${ }^{12,17}$ However, ablation in drops and jets generates complex hydrodynamic phenomena ${ }^{10,11}$ that can rarely be modelled in detail. ${ }^{12}$

At much shorter wavelengths, femtosecond X-ray pulses with very high fluences are now available at X-ray free-electron lasers (XFELs), ${ }^{18,19}$ and can heat matter isochorically to energy densities hundreds of times larger than those required for vaporization of liquid or solid matter. ${ }^{20}$ Here, we present an experimental investigation of XFEL-induced explosions in water drops and jets. Drop and jets are basic liquid systems; also, they are used to deliver samples in many XFEL scattering and imaging experiments. ${ }^{21-23}$ XFEL explosions led to simpler hydrodynamic phenomena than those observed during laser ablation at optical wavelengths. We modelled these phenomena to predict the adverse effects of explosions in XFEL experiments. The relative simplicity of XFEL explosions makes them a promising method for generating far-fromequilibrium conditions, and for triggering phenomena such as phase transitions in XFEL studies. 


\section{XFEL energy deposition mechanism and explosion thresholds}

Fig. 1a shows the geometry of the experiment, performed at the Coherent X-ray Imaging (CXI) instrument ${ }^{24,25}$ at $\operatorname{LCLS}^{18}: 30 \mathrm{fs}$ pulses of $8.2 \mathrm{keV}$ X-ray photons, focused to $\sim 1 \mu \mathrm{m}$ FWHM, intersected water jets (3-20 $\mu \mathrm{m}$ diameter) and drops (32-45 $\mu \mathrm{m}$ diameter) flowing in a vacuum chamber. For each XFEL pulse we imaged optically the explosion once, at a variable time delay after the pulse.

$\mathrm{X}$-ray photons at $8.2 \mathrm{keV}$ are predominantly absorbed on a sub-femtosecond timescale through the photoelectric effect. ${ }^{26,27}$ This generates photoelectrons, which transfer their energy first to other electrons, ${ }^{28}$ and then to water molecules, in a thermalization process expected to take a few picoseconds. ${ }^{29,30}$ In our experiments, the X-ray heating was approximately constant along the illuminated region, because the attenuation length at $8.2 \mathrm{keV}$ photons in water $(\sim 1 \mathrm{~mm})^{26}$ is much longer than the diameter of the jets or drops, and because the absorption of X-rays remains linear up to the highest peak light intensities we used $\left(\sim 3 \cdot 10^{18} \mathrm{~W} / \mathrm{cm}^{3}\right)$. The femtosecond XFEL pulses thus heated isochorically a filament of water inside the jets and drops (Fig. 1b). The volume of this filament can be larger than the illuminated volume because photoelectrons with energies on the order of $10 \mathrm{keV}$ may diffuse over micron distances in materials. ${ }^{31}$ However, at typical XFEL fluences the electric charge of the positive ions generated during absorption traps the photoelectrons within the illuminated volume. ${ }^{28}$ Here, we calculated the isochoric energy density deposited by X-rays assuming that the filament volume was equal to the illuminated volume, and that the X-ray beam had a nominal cross-sectional area of $1 \mu \mathrm{m}^{2}$.

We found that as the pulse energy was increased, the onset of explosions in water coincided with the onset of electrostatic trapping of photoelectrons. We estimated that in our experiments photoelectron trapping requires deposited energy densities larger than $\sim 30 \mathrm{MJ} / \mathrm{kg}$ (see Supplementary Information (SI) for details). The first visible disruptions in liquid were gas bubbles, at a deposited energy density of $\sim 10 \mathrm{MJ} / \mathrm{kg}$; this value is significantly higher than that needed to fully vaporize room-temperature water $(\sim 2.6 \mathrm{MJ} / \mathrm{kg})$, and indicates that the photoelectrons heated a larger volume of water to a lower energy density. Explosions, which we define as the ejection of material from jets or drops, occurred for deposited energy densities above $20-50 \mathrm{MJ} / \mathrm{kg}$. 
The XFEL pulses thus generated a micron-wide "hot" filament of water spanning the entire liquid sample, with an approximately constant energy density ranging from $\sim 50$ to $\sim 750 \mathrm{MJ} / \mathrm{kg}$. To our knowledge, such conditions have never been achieved in laser ablation studies. For example, visible and infrared light pulses are focused by water drops, creating hot spots where the deposition of energy is greatly enhanced by focusing and by nonlinear absorption. ${ }^{11}$

\section{The dynamics of XFEL explosions in drops}

We imaged the XFEL explosions in drops and jets at delays from $5 \mathrm{~ns}$ to hundreds of microseconds after the arrival of X-rays. The explosion patterns were reproducible, allowing the analysis of the explosion dynamics as a function of the pulse energy and time delay, and the recording of explosion movies (available as Supplementary Videos).

Drops intercepted by XFEL pulses fragmented into small liquid particles and vapour, except for a thin film of liquid from the periphery of the drop, and the explosions pushed neighbouring drops towards the next-neighbour drops (Fig 1a). The material from exploded drops expanded approximately perpendicular to the X-ray beam forming a disk-shaped cloud (Fig. 1b-c). In the inertial frame of reference moving with the undisturbed liquid, the explosions were symmetric relative to the illuminated area if the X-ray beam intersected the centre of the drops, and the cloud expanded with a uniform peripheral velocity $v_{\text {gas }}$ (Fig. 1c).

The expansion velocity $v_{\text {gas }}$ quantifies the conversion of energy deposited by light into mechanical energy. A common mechanical effect of the absorption of light pulses generated by optical lasers is the generation of pressure and shock waves. ${ }^{7,32}$ The generation of pressure waves occurs through mechanisms that depend on the properties of the light pulse. At visible wavelengths water is transparent, but strong absorption can occur for femtosecond to nanosecond pulses through nonlinear effects that lead to ionization, and the pressure waves are generated by a high-pressure plasma bubble. ${ }^{6,8}$ In the mid-infrared (approximately 2.5 to $11 \mu \mathrm{m}$ wavelength), water absorbs light strongly and can be heated through linear absorption to temperatures higher than its critical point, ${ }^{9,13,33}$ in which case pressure waves can be generated by thermoelastic effects or by vaporization. Our drop explosion measurements did not probe explosions at the time delays needed to observe possible pressure and shock waves or the mechanism that would 
lead to their generation; however, estimates of the energy fluxes propagating in the drops, detailed in the SI, indicate that shock waves were generated in the drops.

Shock waves transport and redistribute energy, and they also provide a mechanism for generating the motion of liquid. Due to the conservation of mass, momentum, and energy, a pressure or shock wave accelerates the material subjected to the pressure $P_{S}$ to a particle velocity $u_{P}$. If a shock wave reaches an interface with vacuum, the pressure is released as the material is accelerated to approximately twice the particle velocity, $2 u p .{ }^{34}$ The relation between $u P$ and $P_{S}$ is a thermodynamic property, and is known for water. ${ }^{35,36}$

We modelled the explosion of drops assuming that the hot filament produced by X-rays generates a cylindrical shock wave that propagates through the rest of the drop. Since the particle velocity has the same direction as the propagation of the pressure, a cylindrical shock leads to particle velocities oriented in a plane perpendicular to the X-ray beam, and to an explosion pattern that is also perpendicular to the beam. In principle, the shock wave can be modelled in detail, but we found that the scaling of the explosion velocity with the pulse energy can be found using arguments based on average values and on taking into account the cylindrical directionality of the shock.

The pressure generated in drops during explosions can be estimated from the deposited energy density. The thermal pressure in a material is equal to the thermal energy density multiplied by the Grüneisen coefficient or parameter $\Gamma$ ( $\Gamma=0.5$ for liquid water at densities close to 1 $\left.\mathrm{g} / \mathrm{cm}^{3}\right) .{ }^{34,37} \mathrm{We}$ defined an average reference pressure inside the whole drop, $P_{S r}$, assuming that all the energy deposited by X-rays was redistributed uniformly within the drop. The energy absorbed from X-rays is approximately $2 E_{X r a y} \alpha$ Xray $R$, where $E_{\text {Xray }}$ is the pulse energy, $\alpha_{\text {Xray }}$ the $\mathrm{X}$ ray absorption coefficient, and $R$ the drop radius. The energy density after redistribution is $2 E_{X r a y} \alpha_{X r a y} R /\left(4 \pi R^{3} / 3\right)$, which corresponds to a reference pressure $P_{S r}$ given by:

$P_{S r}=\Gamma \frac{3 E_{\text {Xray }} \alpha_{\text {Xray }}}{2 \pi R^{2}}$

To predict the drop explosion velocity, we used the reference pressure $P_{S r}$ in the formula for shock release velocity, $2 u p\left(P_{S}\right)$. Fig. 2 shows the comparison between the measured cloud expansion velocity, $v_{g a s}$, and the shock release velocity in water, $2 u P\left(P_{S r}\right) .{ }^{36}$ The relation $v_{g a s}=$ 
$2 u_{P}\left(P_{S r}\right)$ approximates the explosion velocities well, and we also used it to model the change in the velocity of the neighbouring water drops due to explosions. Assuming an expansion perpendicular to the X-ray beam and a uniform density of the expanding cloud, the change in velocity can be predicted within a factor of two of the measurements (see SI).

\section{The dynamics of XFEL explosions in jets}

In jets, XFEL-induced explosions initially vaporized a section with a size comparable to the jet diameter. This initial gap grew as the jet ends emitted a thin liquid film, which later evolved into an approximately conical shape and folded back until it coalesced with the jet (Fig. 4a). Fig. 4b shows the gap growth in a 20- $\mu \mathrm{m}$ diameter free water jet for several pulse energies. The growth of the gap had three stages: a first stage (I) characterized by decreasing velocities, followed by two stages of growth (II and III) at approximately constant but different velocities. For a given jet diameter, the gap growth rate depended on the pulse energy only during stage I, which is the only stage driven directly by explosions.

Stages II and III are influenced by the surface tension of the liquid jet. Stage III occurs after the coalescence of the film with the jet, and represents the same phenomenon that would occur at the end of a suddenly cut jet. The (half) velocity of gap growth during stage III is equal within experimental accuracy to the jet retraction velocity $v_{\gamma}=2 \sqrt{\gamma_{L} / \rho_{L} R_{j}}$, where $\gamma_{L}$ is the surface tension of water, $\rho_{L}$ the density of water, and $R_{j}$ the jet radius. This formula reflects the conversion of interfacial energy into kinetic energy of the flow. ${ }^{38}$ Stage II is a more complicated case of interfacially-driven flow than stage III, because the jet ends are connected to the liquid films.

Stage I is characterized by the generation of the water films. Thin liquid films can be generated by impinging a liquid jet on a solid stop, ${ }^{39}$ but our liquid films are generated through a liquid-gas interaction and have a dynamic shape. Fig. 5a shows the relevant features of this process as a section of the jet approximately as long as its diameter vaporizes and starts to expand. The vapour applies pressure on both ends of the jet, and pushes liquid from the jets at an approximately right angle to the jet axis. The rate at which liquid flows away from the jet, and thus the velocity at which the jet ends retract, is determined by the balance between the applied pressure and the rate at which the liquid acquires linear momentum when it becomes part of the 
film. The velocity of the gap growth decays in stage I because the vapour cloud expands and its pressure drops rapidly.

The stage I gap growth mechanism described above can be modelled with a logarithmic growth of the gap, starting from a gap length equal to the jet diameter (see the SI for derivation):

$X_{\text {retraction }}=R_{j}+C \cdot R_{j} \ln (1+t / \tau)$

where $X_{\text {retraction }}$ is half of the gap size, $C$ is a numerical constant close to unity, and $t$ is the delay time. The gap growth has a characteristic length scale given by $R_{j}$, and a characteristic timescale $\tau=R_{j} / v_{\text {gas }}$

\section{Predicting the limitations imposed by jet gaps in XFEL experiments}

XFEL explosions in jets remove the jetting liquid, including any samples carried by it, from the interaction region with X-rays. This condition is temporary, because the gap moves with the jet, and the upstream end of the jet will eventually reach the interaction region. The jets we investigated recovered after delays in the microsecond range (see SI), and would not allow full use of X-ray pulses at future $\mathrm{MHz}$ repetition rate facilities such as the European XFEL and LCLS-II. ${ }^{40,41}$

To evaluate the impact of gap formation in XFEL experiments, we derived detailed analytical formulae for the gap dynamics during stage I and its size at the end of stage I, as a function of the properties of the jet and of the XFEL pulse (see SI for derivation). These formulae are based on two approximations of the empirical relation ${ }^{36} P_{S r}=\rho_{L}\left(c_{0}+2 u_{P}\right) u_{P}$, where $c_{0}$ is the speed of sound in water. One is a low pressure regime in which $u_{P} \propto P_{S r}\left(P_{S r}<1 \mathrm{GPa}\right)$, and the other a high pressure regime in which $u_{P} \propto \sqrt{P_{S r}}\left(P_{S r}>10 \mathrm{GPa}\right)$.

The dynamics of the gap in the low $(l)$ and high $(h)$ pressure regimes is estimated using linear momentum arguments with an assumption of cylindrical symmetry and is given by:

$$
X_{\text {retraction }, l}(t)=R_{j}+R_{j} \sqrt{\frac{\rho_{L} c_{0}^{2}}{\delta_{L}}} \frac{\sqrt{K_{E, l}}}{K_{v, l}} \cdot \ln \left(1+\frac{t}{\tau_{l}}\right)
$$

$\tau_{l}=\frac{2}{3} \frac{R_{j}^{3}}{K_{v, l}} \frac{\pi \rho_{L} c_{0}}{E_{\text {Xray }} \alpha_{\text {Xray }} \Gamma}$ 


$$
X_{\text {retraction }, h}(t)=R_{j}+\frac{R_{j}}{\sqrt{2}} \frac{\sqrt{K_{E, h}}}{K_{v, h}} \cdot \ln \left(1+\frac{t}{\tau_{h}}\right)
$$

$\tau_{h}=\frac{R_{j}^{2}}{K_{v, h}} \sqrt{\frac{\pi \rho_{L}}{3 E_{\text {Xray }} \alpha_{\text {Xray }} \Gamma}}$

where $\delta_{L}$ is the cohesive energy density of water $(2.3 \mathrm{GPa})$. We determined, by fitting the measurements, the numerical constants $K_{E, l}=0.08, K_{v, l}=0.21, K_{E, h}=0.06$, and $K_{v, h}=0.12$. These constants account empirically for the fact that some of the deposited energy was used to drive shock waves that travelled along the jets; they would be equal to 1 if all the energy deposited by X-rays were available to drive the gap growth.

Eqs. (3) to (6) predict quantitatively the gap expansion dynamics during stage I (see Fig. 5b and the SI), and also the different scaling of the gap size with the jet size and pulse energy in the two pressure regimes. The different scaling arises due to the different dependence of $u_{P}$ on $P_{S r}$, and due to a partial conversion of the exploded liquid to vapour in the low pressure regime.

The size of the gap at the end of stage I is a useful measure of the damage induced by X-rays in the jet, because the gap growth rate is much slower afterwards. Stage I ends when the retraction rate caused by the jet's surface energy becomes larger than the retraction rate caused by the decaying gas pressure of the cloud. Thus, we find that the (half) gap sizes at the end of stage I, $X$, are given by:

$X_{I, l}=R_{j}+R_{j} \sqrt{\frac{\rho_{L} c_{0}^{2}}{\delta_{L}}} \frac{\sqrt{K_{E, l}}}{K_{v, l}} \cdot \ln \left(\frac{1}{2 \pi} \sqrt{\frac{K_{E, l}}{\gamma_{L} \delta_{L}}} \frac{E_{\text {Xray }} \alpha_{\text {Xray }} \Gamma}{R_{j}^{3 / 2}}\right)$

$X_{I, h}=R_{j}+\frac{R_{j}}{\sqrt{2}} \frac{\sqrt{K_{E, h}}}{K_{v, h}} \cdot \ln \left(\sqrt{\frac{K_{E, h}}{8 \pi} \frac{3 E_{\text {Xray }} \alpha_{\text {Xray }} \Gamma}{\gamma_{L} R_{j}}}\right)$

The gap size at the end of the stage I depends logarithmically on the pulse energy, increasing in size by a length close to the jet diameter when the pulse energy is doubled (see Fig. $4 \mathrm{~b}$ and the SI). Fig. 5c shows the comparison between measurements and Eqs. (7) and (8).

The formulae listed in this section should be applicable to aqueous jets carrying samples that do not lead to a large change in the density, viscosity, or X-ray absorption of the liquid. For 
example, we found that jets of aqueous suspensions (up to $10 \% \mathrm{v} / \mathrm{v}$ ) of protein crystals had the same gap dynamics as jets of pure water (see the SI).

\section{XFEL-induced trains of shock waves in jets}

We observed well-defined shock waves traveling along 20- $\mu$ m diameter jets after explosions induced by $0.75 \mathrm{~mJ}$ X-ray pulses (Fig. 6). Close to the explosion site, the shock velocity was supersonic at $\sim 2500 \mathrm{~m} / \mathrm{s}$. Later during propagation, the shock waves slowed down to a value indistinguishable experimentally from the speed of sound in water $(\sim 1500 \mathrm{~m} / \mathrm{s})$. During the supersonic propagation of the shock, the shock velocity corresponds ${ }^{35}$ to a shock pressure of 1.2 $\mathrm{GPa}$, close to the value of $P_{S r}$ in this experiment $(1.6 \mathrm{GPa})$.

After propagating for tens of nanoseconds, the initial shock split to form up to 6 distinct fronts separated by distances on the order of $10 \mu \mathrm{m}$ (Fig. 6b). This splitting indicates the presence of nanosecond pressure and density oscillations in the liquid after the passage of the first shock. Since the pressure in the jets (equal to the Laplace pressure) is negligible relative to the peak positive pressures generated by the shocks, pressure oscillations can reach negative values (or tension) at which liquid water is stretched and provides a driving force for the next oscillation. The images of shocks provide evidence for such oscillations. The thickness of the dark edge of the jet increases with the refractive index of the liquid, and thus with its density. Fig. 6c shows both positive and negative variations in this thickness, corresponding to densities both above and below the density of water inside the undisturbed jet.

Three-dimensional shocks produced by focused acoustic waves in liquid water can also be followed by an oscillation to negative pressures, but the negative swing is damped by cavitation on a microsecond time scale, and further oscillations are suppressed. ${ }^{42}$ In our case the pressure oscillations occurred on a nanosecond time scale, which should minimize the role of cavitation and allow several positive-to-negative pressure oscillations.

\section{Outlook}

The dynamics of XFEL explosions in drops and jets is related to that encountered in laser ablation, but without the complications of light refraction and nonlinear absorption. Here we 
have shown that the slowest class of explosion effects - the hydrodynamic flows - can be rationalized and predicted quantitatively using analysis based on conservation laws.

Further experimental and modelling work is needed to fully understand the dynamics of XFEL explosions, especially on time scales shorter than a few nanoseconds. Given the reproducibility and symmetry of the hydrodynamic phenomena, it is reasonable to expect that phenomena occurring earlier, such as the vaporization of the liquid, are also reproducible and controllable. More generally, XFEL explosions might provide methods to control the states of matter not only at the high energy density conditions produced shortly after the absorption of X-rays, ${ }^{20}$ but also at lower energy scales that are currently inaccessible experimentally.

For example, the train of shocks observed in jets show that XFEL explosions can apply large transient pressures in liquids on nanosecond time scales. These transient pressures are both a problem and an opportunity, because they can change rapidly the environment (such as $\mathrm{pH}^{43}$ ) or the structure ${ }^{44}$ of samples probed at XFEL facilities. We believe that the most promising application of XFEL explosions is to trigger processes that depend on pressure, and then probe these processes with a second XFEL pulse, using recently developed pump-and-probe methods based on dual XFEL pulses ${ }^{45,46}$ or on split-and-delay lines. ${ }^{47}$ Such experiments may reveal, with unprecedented spatial and temporal resolution, the structure and dynamics of pure liquids and of chemical or biological samples in solution.

\section{Methods}

This section contains a brief account of the experimental and data analysis methods. A detailed description of the methods and of the explosion models is provided in the Supplementary Information.

Experimental setup. The experimental system is shown in Fig. 1a. The experiments were performed at the Coherent X-ray Imaging ${ }^{24,25}$ (CXI) endstation at LCLS, in a vacuum chamber at pressures of 1-10 mTorr. We injected liquid drops and jets into the path of X-ray laser pulses, and we imaged optically the explosions approximately perpendicular to the direction of propagation of X-rays, using a high-resolution imaging system built for this experiment. Speckle-free time-resolved brightfield illumination was provided by $800 \mathrm{~nm}$ femtosecond laser pulses (Coherent Inc., Legend Elite) decohered through propagation in optical fibers and through 
scattering on diffusers. The high-resolution images were recorded with a high-speed camera (Vison Research, Miro M320S). To record the inline image shown in Fig. 2c, we used the existing low-resolution inline monitoring system at CXI with brightfield femtosecond illumination.

Materials. We investigated drops and jets of pure water (EMD Millipore, Milli-Q Integral 3), and jets of aqueous suspensions of ferritin crystals (5-7 $\mu \mathrm{m}, 10 \pm 2 \% \mathrm{v} / \mathrm{v})$ and apoferritin crystals (3-4 $\mu \mathrm{m}, 8 \pm 2 \% \mathrm{v} / \mathrm{v})$.

X-ray laser parameters. The experiments were carried out with XFEL pulses produced at a rate of $120 \mathrm{~Hz}$. The X-ray photon energy was $8.2 \mathrm{keV}$, except for the two-axis imaging experiment shown in Fig. 2c, which used $9.5 \mathrm{keV}$ X-rays. The pulses had $\sim 2 \mathrm{~mJ}$ total energy at the source, and were attenuated to a desired amount before reaching the sample; the energy of each pulse was measured after generation. The X-rays were focused to $\sim 1 \mu \mathrm{m}$ FWHM spot, corresponding to a Rayleigh length $(\sim 2 \mathrm{~cm})$ much longer than the diameter of the drops and jets. We used previously calibrated values of the beam width, and of beamline transmission losses between the source and CXI, to calculate the energy deposited in drops and jets.

Production of drops and jets. We produced drops synchronized to the X-ray pulses using piezoelectric nozzles (MicroFab Technologies, Inc.) to induce the Rayleigh-Plateau breakup of free water microjets. Jets were produced using either the piezoelectric nozzles without a driving signal, or gas-focused GDVN injectors. ${ }^{48}$

Data acquisition and processing. We carried out the experiments as separate runs, for each set of drop or jet size and a fixed attenuation of the XFEL pulses. All raw data was filtered to remove pulses that had large variations from the average pulse energy in the run. The data for gas-focused jets was also filtered to remove shots on jets poorly aligned with the X-rays. We determined the kinematics of explosions from the recorded images, and we wrote Matlab codes to extract automatically the size of the gap in jets.

Supplementary videos. We assembled movies of explosions at equivalent frame rates between 2.5 and 200 million frames per second, from single-shot images of explosions. The individual images were filtered according to the same criteria used for the data acquisition. The images in 
the movies were shifted to compensate for camera vibrations. We did not alter the contrast or brightness of the original images.

Modeling. The explosion models are analytical, and were developed to provide the scaling of explosions with the liquid parameters and with XFEL pulse properties, as well as quantitative predictions in the form of algebraic formulas.

Data Availability Statement (DAS). Raw data were generated at the Linac Coherent Light Source (LCLS) at the SLAC National Accelerator Laboratory. Complete image data sets and corresponding metadata supporting the findings of this study are available ${ }^{49}$ from http://purl.stanford.edu/wv179nv3100

\section{Acknowledgments}

The work was primarily supported by the in-house research program at the Linac Coherent Light Source (LCLS) at the SLAC National Accelerator Laboratory, and by the U.S. Department of Energy, Office of Science, Chemical Sciences, Geosciences, and Biosciences Division. Use of the Linac Coherent Light Source (LCLS), SLAC National Accelerator Laboratory, is supported by the U.S. Department of Energy, Office of Science, Office of Basic Energy Sciences under Contract no. DE-AC02-76SF00515. S. Botha, K.N., I.S., and R.L.S. acknowledge support from the Max Planck Society. The development of the optical imaging setup was partially supported by the Human Frontiers Science Project Award RPG005/2011, and by the SLAC Laboratory Directed Research and Development Program. We thank Robin Curtis for his assistance in assembling the experiment and Stefan Hau-Riege for discussions.

\section{Author contributions}

C.A.S. and S. Boutet conceived the experiment; C.A.S., D.M., R.G.S., T.A.M., M.M., G.J.W., J.E.K., M.J.H., S.A.H.G., J.S.R., K.L.G., S. Botha, K.N., I.S., R.L.S. and S. Boutet designed, developed, and built the experimental apparatus; C.A.S., H.L., M.M., G.J.W., J.E.K., M.L., A.L.A., P.R.W., S. Botha, K.N., I.S., R.L.S., and S. Boutet carried out the experiments; C.A.S., H.L., and T.J.L. processed the data; C.A.S., D.M., and H.A.S. interpreted the data; C.A.S. 
developed the fluid dynamics models; C.A.S., D.M., I.S., R.L.S., H.A.S., and S. Boutet wrote the paper.

\section{Additional information}

302 Supplementary information is available in the online version of the paper. Correspondence and requests for materials should be addressed to C.A.S. 


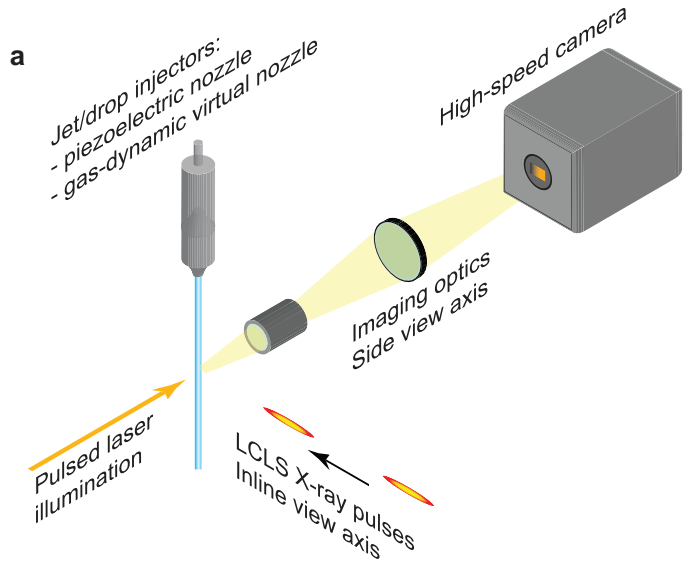

b

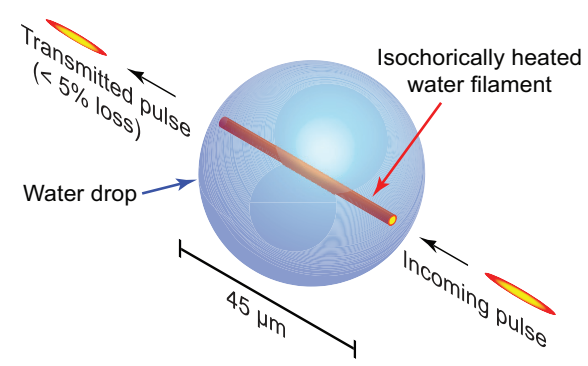

Figure 1 | Inducing liquid microexplosions with ultraintense $\mathbf{X}$-ray pulses. a, Experimental design. Pulses from an X-ray free-electron laser, focused to a $\sim 1 \mu \mathrm{m}$ beam diameter, intercept water droplets ( 32 to $45 \mu \mathrm{m}$ diameter) or water jets (2.75 to $30 \mu \mathrm{m}$ diameter) flowing in a vacuum chamber. The subsequent phenomena are imaged optically, after a variable delay time, using a femtosecond laser for illumination. b, Schematic of the energy deposition process. A focused femtosecond X-ray pulse passes undeflected though the drops, and a small fraction of the pulse energy is absorbed in the drop, heating isochorically a micron-wide filament of water to average energy densities tens to hundreds of times larger than those needed to vaporize water. 
b
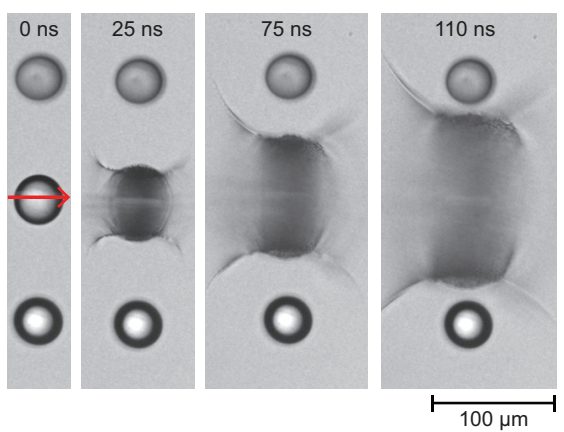

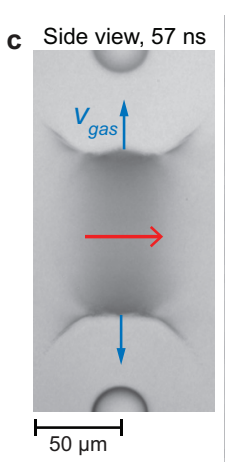

Inline view, $57 \mathrm{~ns}$

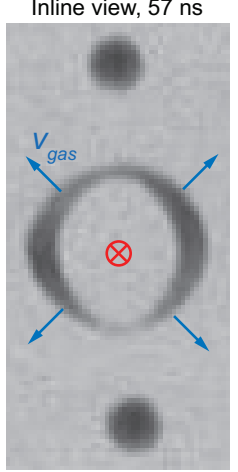

Figure 2 | Drop explosions induced by XFEL pulses. a, Explosions induced by $0.75 \pm 0.08 \mathrm{~mJ}$, $8.2 \mathrm{keV}$ X-ray pulses in a train of $40-\mu \mathrm{m}$ diameter drops. A drop explodes, and accelerates the neighbour drops (blue arrows) towards the next-neighbour drops (orange arrows) until they collide and coalesce (green arrows). b, High-resolution images of drop explosions for the same parameters as in panel a. Water drops fragment into a cloud that expands perpendicular to the X-ray path. c, Three-dimensional symmetry of drop explosions ( $32 \mu \mathrm{m}$ diameter drop, $1 \pm 0.1 \mathrm{~mJ}$ pulse, $9.5 \mathrm{keV}$ $\mathrm{X}$-rays). Two images, taken along the X-ray beam and perpendicular to it, show that the expanding cloud is disk-shaped and bounded by a liquid film. If the X-rays are aligned with the centre of the drops, the expansion is symmetric and has a peripheral velocity $v_{\text {gas }}$. 


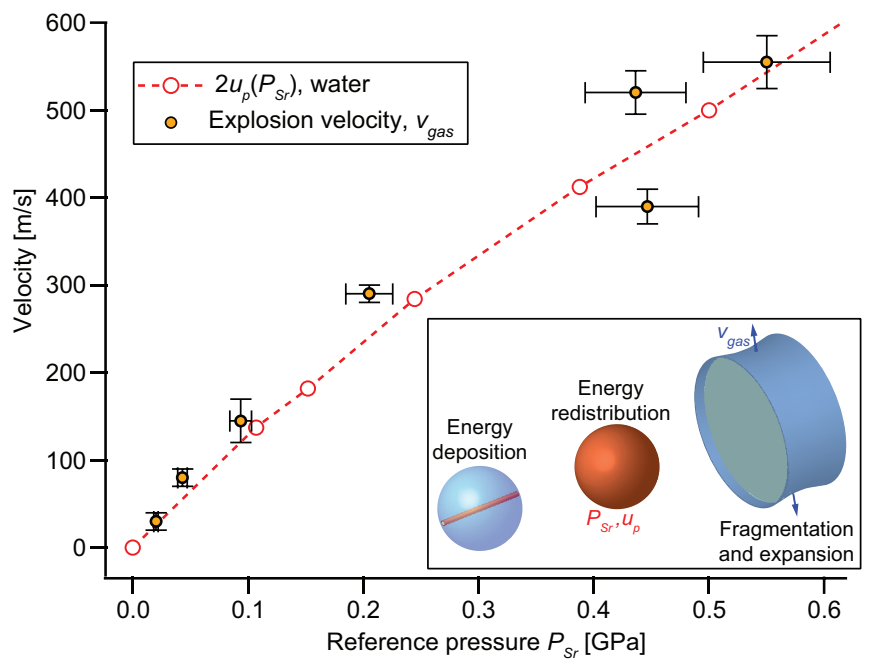

Figure 3 | Quantitative dynamics of drop explosions. The expansion velocity vgas can be estimated using the simplified mechanism depicted in the inset. The water illuminated by X-rays acquires high pressures and launches a pressure wave that redistributes the absorbed energy in the whole drop, leading to an average pressure $P_{S r}$ (Eqn. (1)) and a corresponding particle velocity $u p$; the drop then fragments and expands at a velocity $v_{g a s}=2 u_{P}$. The graph compares $v_{g a s}$ from water drop explosions $(32,40$, and $45 \mu \mathrm{m}$ drop diameters; 8.2 and $9.5 \mathrm{keV}$ X-ray pulses, at several energies) with the shock release velocity $2 u_{P}$ in water. The error bars represent the uncertainty of the measurements. 


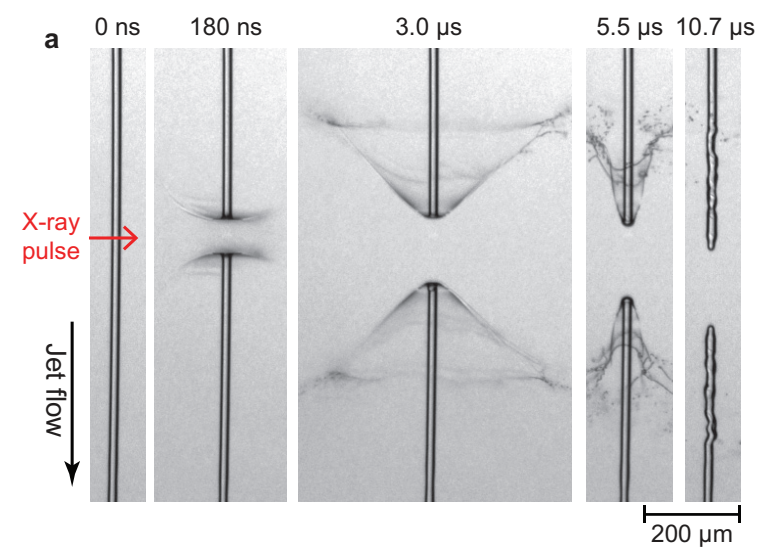

Figure 4 | Jet explosions induced by XFEL pulses. a, Images of explosions induced by $0.75 \pm 0.08$ mJ, $8.2 \mathrm{keV}$ XFEL pulses in a 20 - $\mu \mathrm{m}$ diameter water jet. A gap forms after a jet section near the X-ray spot vaporizes explosively. Liquid from the jet ends is then pushed into thin conical films of water, which later collapse onto the jet. b, The gap dynamics. The graph shows the evolution of the gap size in $20-\mu \mathrm{m}$ water jets, including the ones shown in panel a. The gap has a logarithmic growth stage (I), followed by two stages of linear growth (II and III). The growth rate depends on the pulse energy only during stage I. The error bars represent the uncertainty of measurements. 

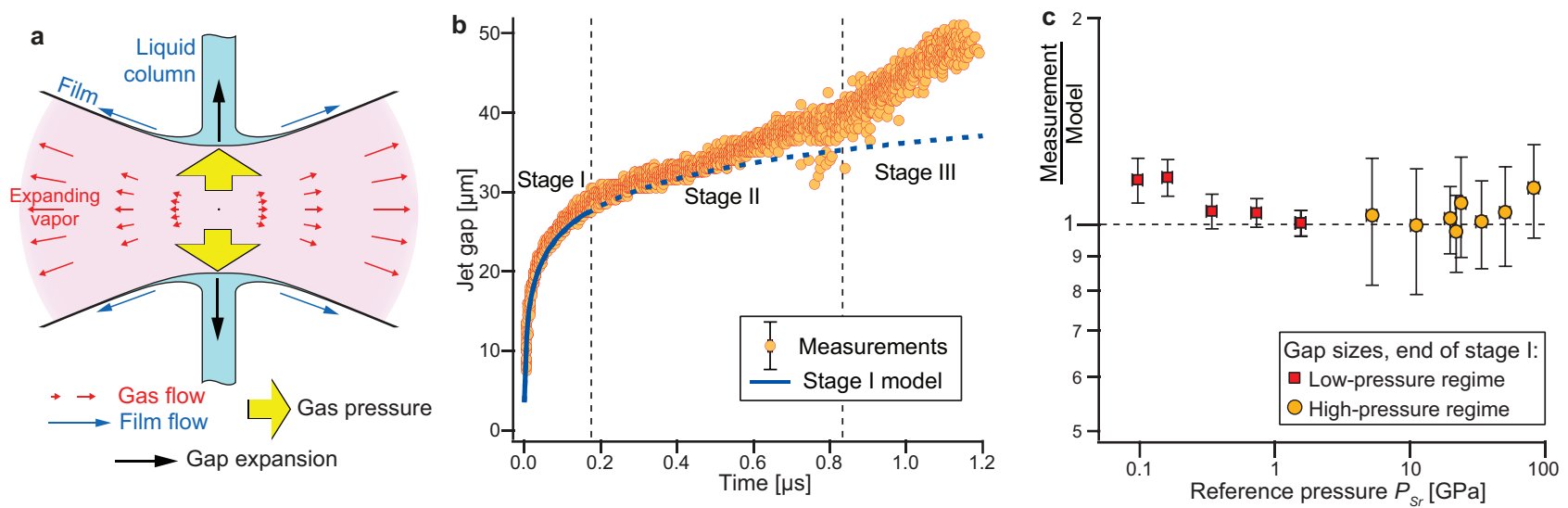

Figure 5 | A model for the vapour-driven growth of the gap. a, Mechanism of gap growth during stage I. The pressure inside the expanding cloud of vaporized material pushes liquid from the intact regions of the jet into thin films; the intact jet sections are consumed at a velocity that depends on the pressure inside the cloud. b, Model of gap growth during stage I (Eqs. (5) and (6), blue line) and experimental data on a 3.5- $\mu \mathrm{m}$ diameter jet (orange circles). c, The size of the gap at the end of stage I can be modeled in two regimes of $P_{S r}$ pressures. The graph compares the model (Eqs. (7) and (8)) with the experimental data, for all jets. The error bars represent the uncertainty of experimental data. 

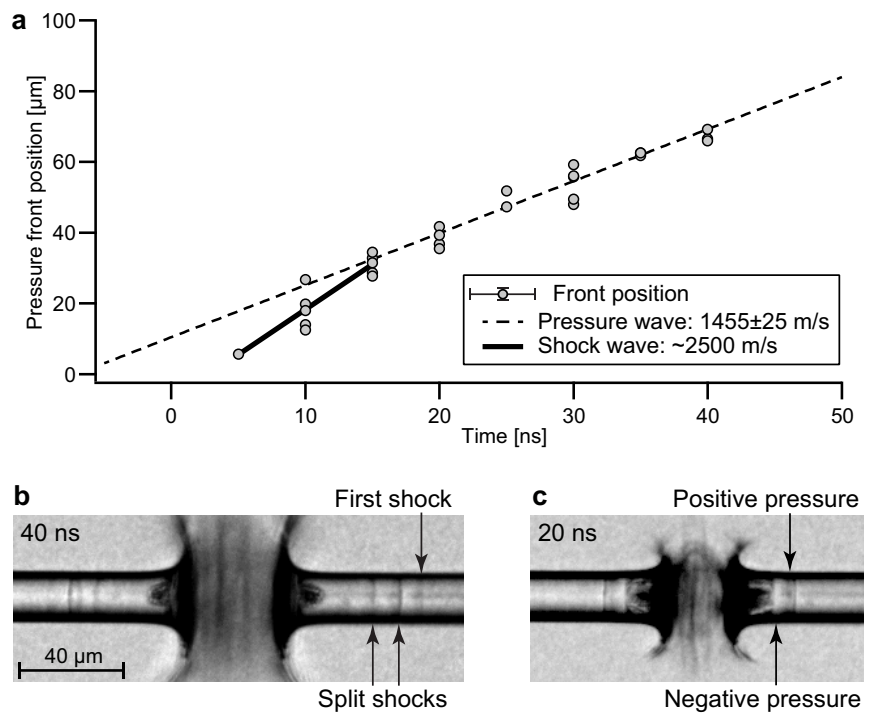

Figure 6 | The propagation of XFEL-induced shock waves in jets. a, The shock waves launched in a $20-\mu \mathrm{m}$ diameter jet initially propagate at supersonic velocities and then slow down to the speed of sound. $\mathbf{b}$, Multiple shock waves split from the first one during propagation. The data shown in panel a is for the propagation of the first shock. c, Regions where the density of liquid water is higher or lower than in the undisturbed jet are visible as changes in the thickness of the dark edge of the jet. Thicker edges indicate higher densities and positive pressures; thinner edges indicate lower densities and negative pressures. 


\section{References}

1 Burrows, A. Supernova explosions in the Universe. Nature 403, 727-733 (2000).

2 Vogel, A. \& Venugopalan, V. Mechanisms of Pulsed Laser Ablation of Biological Tissues. Chem. Rev. 103, 577-644 (2003).

3 Vailionis, A. et al. Evidence of superdense aluminium synthesized by ultrafast microexplosion. Nat. Commun. 2, 445 (2011).

4 Sokolowski-Tinten, K. et al. Transient States of Matter during Short Pulse Laser Ablation. Phys. Rev. Lett. 81, 224-227 (1998).

5 Milathianaki, D. et al. Femtosecond Visualization of Lattice Dynamics in ShockCompressed Matter. Science 342, 220-223 (2013).

6 Schaffer, C. B., Nishimura, N., Glezer, E. N., Kim, A. M. T. \& Mazur, E. Dynamics of femtosecond laser-induced breakdown in water from femtoseconds to microseconds. Opt. Express 10, 196-203 (2002).

7 Vogel, A., Busch, S. \& Parlitz, U. Shock wave emission and cavitation bubble generation by picosecond and nanosecond optical breakdown in water. J. Acoust. Soc. Am. 100, 148165 (1996).

8 Vogel, A., Linz, N., Freidank, S. \& Paltauf, G. Femtosecond-Laser-Induced Nanocavitation in Water: Implications for Optical Breakdown Threshold and Cell Surgery. Phys. Rev. Lett. 100, 038102 (2008).

9 Charvat, A., Stasicki, B. \& Abel, B. Product Screening of Fast Reactions in IR-LaserHeated Liquid Water Filaments in a Vacuum by Mass Spectrometry. J. Phys. Chem. A 110, 3297-3306 (2006).

10 Zhang, J. Z., Lam, J. K., Wood, C. F., Chu, B. T. \& Chang, R. K. Explosive vaporization of a large transparent droplet irradiated by a high-intensity laser. Appl. Opt. 26, 47314737 (1987).

11 Lindinger, A. et al. Time-resolved explosion dynamics of $\mathrm{H}_{2} \mathrm{O}$ droplets induced by femtosecond laser pulses. Appl. Opt. 43, 5263-5269 (2004).

12 Klein, A. L. et al. Drop Shaping by Laser-Pulse Impact. Phys. Rev. Appl. 3, 044018 (2015).

13 Franjic, K. \& Miller, R. J. D. Vibrationally excited ultrafast thermodynamic phase transitions at the water/air interface. PCCP 12, 5225-5239 (2010).

14 Strycker, B. et al. Femtosecond-laser-induced shockwaves in water generated at an airwater interface. Opt. Express 21, 23772-23784 (2013).

15 Ando, K., Liu, A. Q. \& Ohl, C. D. Homogeneous Nucleation in Water in Microfluidic Channels. Phys. Rev. Lett. 109 (2012).

16 Vogel, A., Noack, J., Huttman, G. \& Paltauf, G. Mechanisms of femtosecond laser nanosurgery of cells and tissues. Appl. Phys. B 81, 1015-1047 (2005).

17 Kurz, H. G. et al. High-order-harmonic generation from dense water microdroplets. Phys. Rev. A 87, 063811 (2013).

18 Emma, P. et al. First lasing and operation of an ångstrom-wavelength free-electron laser. Nat. Photonics 4, 641-647 (2010).

19 Ishikawa, T. et al. A compact X-ray free-electron laser emitting in the sub-ångström region. Nat. Photonics 6, 540-544 (2012).

20 Vinko, S. M. et al. Creation and diagnosis of a solid-density plasma with an X-ray freeelectron laser. Nature 482, 59-62 (2012). 
21 Chapman, H. N. et al. Femtosecond X-ray protein nanocrystallography. Nature 470, 7377 (2011).

22 Boutet, S. et al. High-Resolution Protein Structure Determination by Serial Femtosecond Crystallography. Science 337, 362-364 (2012).

23 Kern, J. et al. Simultaneous Femtosecond X-ray Spectroscopy and Diffraction of Photosystem II at Room Temperature. Science 340, 491-495 (2013).

24 Liang, M. et al. The Coherent X-ray Imaging instrument at the Linac Coherent Light Source. J. Synchrotron Radiat. 22, 514-519 (2015).

25 Boutet, S. \& Williams, G. J. The Coherent X-ray Imaging (CXI) instrument at the Linac Coherent Light Source (LCLS). New J. Phys. 12, 035024 (2010).

26 Seltzer, S. M. Calculation of Photon Mass Energy-Transfer and Mass Energy-Absorption Coefficients. Radiat. Res. 136, 147-170 (1993).

27 Neppl, S. et al. Attosecond Time-Resolved Photoemission from Core and Valence States of Magnesium. Phys. Rev. Lett. 109, 087401 (2012).

28 Hau-Riege, S. P. Nonequilibrium electron dynamics in materials driven by high-intensity x-ray pulses. Phys. Rev. E 87 (2013).

29 Siwick, B. J., Dwyer, J. R., Jordan, R. E. \& Miller, R. D. An Atomic-Level View of Melting Using Femtosecond Electron Diffraction. Science 302, 1382-1385 (2003).

30 Vodopyanov, K. L. Saturation studies of $\mathrm{H}_{2} \mathrm{O}$ and $\mathrm{HDO}$ near $3400 \mathrm{~cm}^{-1}$ using intense picosecond laser pulses. J. Chem. Phys. 94, 5389-5393 (1991).

31 Sanishvili, R. et al. Radiation damage in protein crystals is reduced with a micron-sized X-ray beam. Proc. Natl. Acad. Sci. U.S.A. 108, 6127-6132 (2011).

32 Sigrist, M. W. Laser generation of acoustic waves in liquids and gases. J. Appl. Phys. 60, R83-R121 (1986).

33 Kudryashov, S. I., Lyon, K. \& Allen, S. D. Photoacoustic study of relaxation dynamics in multibubble systems in laser-superheated water. Phys. Rev. E 73, 055301(R) (2006).

34 Zel'dovich, Y. B. \& Raizer, Y. P. Physics of Shock Waves and High-Temperature Hydrodynamic Phenomena. Vol. 1-2 (Academic Press, 1968).

35 Rice, M. H. \& Walsh, J. M. Equation of State of Water to 250 Kilobars. J. Chem. Phys. 26, 824-830 (1957).

36 Nagayama, K., Mori, Y., Shimada, K. \& Nakahara, M. Shock Hugoniot compression curve for water up to $1 \mathrm{GPa}$ by using a compressed gas gun. J. Appl. Phys. 91, 476-482 (2002).

37 Gurtman, G. A., Kirsch, J. W. \& Hastings, C. R. Analytical Equation of State for Water Compressed to 300 Kbar. J. Appl. Phys. 42, 851-857 (1971).

38 Ranz, W. E. Some Experiments on the Dynamics of Liquid Films. J. Appl. Phys. 30, 1950-1955 (1959).

39 Clanet, C. Waterbells and Liquid Sheets. Annu. Rev. Fluid Mech. 39, 469-496 (2007).

40 Altarelli, M. \& Mancuso, A. P. Structural biology at the European X-ray free-electron laser facility. Philos. Trans. R. Soc. B 369, 20130311 (2014).

41 Schoenlein, R. et al. New Science Opportunities Enabled by LCLS-II X-ray Lasers. SLAC Report SLAC-R-1053 (2015).

42 Pishchalnikov, Y. A. et al. Cavitation selectively reduces the negative-pressure phase of lithotripter shock pulses. Acoust. Res. Lett. Onl. 6, 280-286 (2005).

43 Lown, D., Thirsk, H. \& Wynne-Jones, L. Effect of Pressure on Ionization Equilibria in Water at $25^{\circ}$ C. Trans. Faraday Soc. 64, 2073-2080 (1968). 
44 Weber, G. \& Drickamer, H. G. The effect of high pressure upon proteins and other biomolecules. Q. Rev. Biophys. 16, 89-112 (1983).

45 Decker, F. et al. A demonstration of multi-bunch operation in the LCLS. Proceedings of the 2010 Free Electron Laser Conference, Malmo Sweden (2010).

46 Marinelli, A. et al. High-intensity double-pulse X-ray free-electron laser. Nat. Commun. 6, 6369 (2015).

47 Alonso-Mori, R. et al. The X-ray Correlation Spectroscopy instrument at the Linac Coherent Light Source. J. Synchrotron Radiat. 22, 508-513 (2015).

48 DePonte, D. P. et al. Gas dynamic virtual nozzle for generation of microscopic droplet streams. J. Phys. D: Appl. Phys. 41, 195505 (2008).

49 Stan, C. A. et al. Image data analyzed in "Liquid explosions induced by X-ray laser pulses". (Stanford Digital Repository, 2016). Available at: http://purl.stanford.edu/wv179nv3100 